\title{
Superfluidity and pairing phenomena in ultracold atomic Fermi gases in one-dimensional optical lattices, Part I: Balanced case
}

\author{
Jibiao Wang, ${ }^{1,2}$ Leifeng Zhang, ${ }^{2} \mathrm{Yi} \mathrm{Yu},{ }^{3}$ Chaohong Lee, ${ }^{1,4}$ and Qijin Chen ${ }^{5,2,6, \text { * }}$ \\ ${ }^{1}$ Laboratory of Quantum Engineering and Quantum Metrology, School of Physics and Astronomy, \\ Sun Yat-Sen University (Zhuhai Campus), Zhuhai, Guangdong 519082, China \\ ${ }^{2}$ Department of Physics and Zhejiang Institute of Modern Physics, \\ Zhejiang University, Hangzhou, Zhejiang 310027, China \\ ${ }^{3}$ College of Chemical Engineering, Zhejiang University of Technology, Hangzhou, Zhejiang 310014, China \\ ${ }^{4}$ State Key Laboratory of Optoelectronic Materials and Technologies, \\ Sun Yat-Sen University (Guangzhou Campus), Guangzhou, Guangdong 510275, China \\ ${ }^{5}$ Shanghai Branch, National Laboratory for Physical Sciences at Microscale and Department of Modern Physics, \\ University of Science and Technology of China, Shanghai 201315, China \\ ${ }^{6}$ Synergetic Innovation Center of Quantum Information and Quantum Physics, Hefei, Anhui 230026, China
}

(Dated: January 3, 2020)

\begin{abstract}
The superfluidity and pairing phenomena in ultracold atomic Fermi gases have been of great interest in recent years, with multiple tunable parameters. Here we study the BCS-BEC crossover behavior of balanced twocomponent Fermi gases in a one-dimensional optical lattice, which is distinct from the simple three-dimensional (3D) continuum and a fully 3D lattice often found in a condensed matter system. We use a pairing fluctuation theory which includes self-consistent feedback effects at finite temperatures, and find widespread pseudogap phenomena beyond the BCS regime. As a consequence of the lattice periodicity, the superfluid transition temperature $T_{c}$ decreases with pairing strength in the BEC regime, where it approaches asymptotically $T_{c}=\operatorname{\pi an} / 2 \mathrm{~m}$, with $a$ being the $s$-wave scattering length, and $n(m)$ the fermion density (mass). In addition, the quasi-two dimensionality leads to fast growing (absolute value of the) fermionic chemical potential $\mu$ and pairing gap $\Delta$, which depends exponentially on the ratio $d / a$. Importantly, $T_{c}$ at unitarity increases with the lattice constant $d$ and hopping integral $t$. The effect of the van Hove singularity on $T_{c}$ is identified. The superfluid density exhibits $T^{3 / 2}$ power laws at low $T$, away from the extreme BCS limit. These predictions can be tested in future experiments.
\end{abstract}

\section{INTRODUCTION}

Ultracold atomic Fermi gases loaded in optical lattices have attracted enormous attention in condensed matter and atomic, molecular and optical (AMO) physics [1-3]. With multiple easily tunable parameters, they become more and more important as a quantum simulator nowadays [4-6]. Fermions in pure optical lattices are often described by a Hubbard model [5-7]. Among them, the one-dimensional (1D) case can be solved exactly via the Bethe ansatz [8]. However, while for a 1D Hubbard model, each site has at most two fermions, the 1D optical lattice is actually rather different; each site in the lattice direction corresponds to a 2D plane in the transverse dimensions, and thus can accommodate many fermions. Therefore, a 1D optical lattice is a quasi-2D or 3D system [9, 10], depending on the lattice parameters. Moreover, the genuine 1D Hubbard model does not possess a long-range order, hence it can not support a superfluid phase. In contrast, fermions trapped in 1D optical lattices can not only form a superfluid [11, 12], but also exhibit interesting pseudogap phenomena in the normal state [13]. A condensed matter analogue of the 1D optical lattice is the superlattice of semiconductor heterostructures such as the $\mathrm{AlGaAs} / \mathrm{GaAs} / \mathrm{InGaAs}$ structure, except now we are considering pairing phenomena under a tunable attractive interaction.

\footnotetext{
* Corresponding author: qchen@ zju.edu.cn
}

Including the Hubbard model, there has been extensive literature on 3D (and 2D or 1D) lattices in the field of condensed matter [14, 15]. Most of these existing Hubbard model based works address pure lattice cases, since the kinetic energy term often contains only the lattice site hopping [1, 7, 1620]. The "1D optical lattice" in many theoretical works in the literature was actually a genuine 1D lattice in the traditional sense [21]. Here, following the terminology often used by the experimental community [2], we emphasize that, by $1 D$ optical lattice, we mean a periodic stack of $2 D$ planes, and therefore it is a mix of continuum in the transverse 2D $x y$ planes and lattice discreteness in the longitudinal $z$ direction. Theoretical studies on such a 1D optical lattice have been scarce. Devreese et al. studied possible Fulde-Ferrell-LarkinOvchinnikov (FFLO) states [22, 23] in such a 1D optical lattice [24]. Like many others in the literature [7], when studying population imbalance effects, they use the fermion chemical potential $\mu$ and the chemical potential difference $h$ as control variables. While this choice makes numerical calculations simpler, it often restricts the study to the BCS and crossover regimes. Indeed, the superfluid and pairing physics in a 1D optical lattice has not been adequately studied thus far. Given the various available tuning parameters, including the pairing interaction strength, lattice constant and depth, fermion density, population imbalance, as well as mass imbalance in the case of a Fermi-Fermi mixture, there are certainly many facets of the phase diagram and associated very rich physics. In particular, one would like to know if there are exotic new phases emerging, and how to properly characterize such a 1D optical 
lattice.

In this paper, we study two-component fermions loaded in 1D optical lattices using a pairing fluctuation theory, which has been applied successfully to various BCS-BEC crossover phenomena [4, 25,-27], including in quasi-2D and 3D optical lattices [15, 17, 28]. These systems can be quasi-2D or $3 \mathrm{D}$, depending on the lattice constant $d$ and hopping integral $t$ [29], as well as the pairing strength. Due to the complexity induced by multiple tunable parameters, in this paper (Part I), we restrict ourselves to population (and mass) balanced cases only. Here we consider the combined effects of lattice constant, hopping integral, and interaction strength. We find that the mixing between continuum and discrete lattice dimensions leads to exponential behavior of the fermionic chemical potential $\mu$ and the pairing gap $\Delta$ as a function of $d / a$ in the BEC regime, where $a$ is the two-body $s$-wave scattering length, in contrast to the power laws in the pure 3D continuum or 3D lattice cases. We shall present detailed phase diagrams as the system undergoes the BCS-BEC crossover with different lattice constants and hopping integrals, and mainly focus on the finite temperature effects, especially the pseudogap phenomena [30, 31]. As these phase diagrams reveal, (i) the pseudogap phenomena widely exist; (ii) At unitarity, $T_{c}$ increases with the increase of lattice constant or hopping integral; (iii) As a consequence of the lattice periodicity, $T_{c}$ decreases with pairing strength in the BEC regime, and approaches asymptotically $T_{c}=\pi a n / 2 m$, where $n$ is the atom number density, $m$ the atomic mass; (iv) In addition, the quasi-two dimensionality leads to fast growing (absolute value of the) fermionic chemical potential $\mu$ and pairing gap $\Delta$, which depends exponentially on the ratio $d / a$; (v) Due to the contribution of finite momentum pairs, the temperature dependence of the superfluid density $n_{s} / m$ at low $T$ evolves from exponential in the extreme BCS limit to a simple $T^{3 / 2}$ power law in the $\mathrm{BEC}$ regime, for both the in-plane and the out-of-plane (lattice) components.

\section{THEORETICAL FORMALISM}

\section{A. General theory}

While the in-plane ( $x y$ directions) motion of the fermions has a free parabolic dispersion, we use a one-band nearestneighbor tight-binding model for the out-of-plane lattice dimension ( $z$ direction), with the single particle dispersion given by $\xi_{\mathbf{k} \sigma}=\mathbf{k}_{\|}^{2} / 2 m+2 t\left[1-\cos \left(k_{z} d\right)\right]-\mu_{\sigma} \equiv \epsilon_{\mathbf{k}}-\mu_{\sigma}$. Here $\mathbf{k}_{\|} \equiv\left(k_{x}, k_{y}\right)$ and $t$ is the hopping integral between neighboring lattice sites, $d$ the optical lattice constant and $\mu_{\sigma}$ the fermionic chemical potentials for two (pseudo)spins $\sigma=\uparrow, \downarrow$. In the absence of imbalance, we have $\mu_{\sigma}=\mu$, and $\xi_{\mathbf{k} \sigma}=\xi_{\mathbf{k}}$, and we shall drop the spin indices. We restrict $k_{z}$ to the first Brillouin zone (BZ) $[-\pi / d, \pi / d]$ due to the lattice periodicity and set the volume $V=1, \hbar=k_{B}=1$. The oneband approximation is justified when the band gap is tuned large. The fundamental formalism of the pairing fluctuation theory for the present work is the same as that given in Refs. [4, 25], except that we need to rederive the equations with the continuum-lattice mixed dispersion. To keep this paper self-contained, here we recapitulate the derivation and main equations.

The (inverse) bare Green's function is given by $G_{0}^{-1}(K)=$ $i \omega_{n}-\xi_{\mathbf{k}}$, with the self-energy $\Sigma(K)=\sum_{Q} t(Q) G_{0}(Q-$ $K)$. Following Ref. [25], we use a four-vector notation, $\sum_{K} \equiv T \sum_{n} \sum_{\mathbf{k}}, \sum_{Q} \equiv T \sum_{l} \sum_{\mathbf{q}}$, and $K \equiv\left(i \omega_{n}, \mathbf{k}\right)$, $Q \equiv\left(i \Omega_{l}, \mathbf{q}\right)$, where $\omega_{n}=(2 n+1) \pi T, \Omega_{l}=2 l \pi T$ are odd and even Matsubara frequencies, respectively [32]. At finite $T$, the $T$-matrix $t(Q)$ contains a contribution from condensed pairs $t_{s c}(Q)$ and noncondensed pairs $t_{p q}(Q)$, with $t(Q)=t_{s c}(Q)+t_{p g}(Q)$, where $t_{s c}(Q)=-\left(\Delta_{s c}^{2} / T\right) \delta(Q)$ vanishes for $T>T_{c}$, and $t_{p g}(Q)=U /[1+U \chi(Q)]$, with the short range $s$-wave pairing interaction pairing strength $U<0$ and the pair susceptibility $\chi(Q)=\sum_{K} G_{0}(Q-K) G(K)$. Here $G(K)$ is the full Green's function, with the self-energy given by $\Sigma(K)=\Sigma_{s c}(K)+\Sigma_{p g}(K)$, where $\Sigma_{s c}(K)=$ $\sum_{Q} t_{s c}(Q) G_{0}(Q-K)=-\Delta_{s c}^{2} G_{0}(-K)$, and $\Sigma_{p g}(K)=$ $\sum_{Q} t_{p g}(Q) G_{0}(Q-K)$. At $T \leq T_{c}$, the generalized Thouless criterion [33], or equivalently BEC condition for pairs, requires $t_{p g}^{-1}(Q=0)=U^{-1}+\chi(0)=0$. This implies that $t_{p g}(Q)$ is dominated by the vicinity of $Q=0$, so that $\Sigma_{p g}(K)$ may be approximated by $\Sigma_{p g}(K) \approx \sum_{Q} t_{p g}(Q) G_{0}(-K) \equiv$ $-\Delta_{p g}^{2} G_{0}(-K)$, where $\Delta_{p g}^{2} \equiv-\sum_{Q} t_{p g}(Q)$ and we have discarded the incoherent background part of the self energy. (The parameter $\Delta_{p g}$ is referred to as pseudogap, as is widely found in cuprate superconductors [34]). Then the total selfenergy $\Sigma(K)$ takes the simple BCS-like form, $\Sigma(K)=$ $-\Delta^{2} G_{0}(-K)$, where $\Delta^{2}=\Delta_{s c}^{2}+\Delta_{p g}^{2}$. Finally, the Dyson's equation $G^{-1}(K)=G_{0}^{-1}(K)-\Sigma(K)$ leads immediately to the full Green's function

$$
G(K)=\frac{u_{\mathbf{k}}^{2}}{i \omega_{n}-E_{\mathbf{k}}}+\frac{v_{\mathbf{k}}^{2}}{i \omega_{n}+E_{\mathbf{k}}},
$$

where $u_{\mathbf{k}}^{2}=\left(1+\xi_{\mathbf{k}} / E_{\mathbf{k}}\right) / 2, v_{\mathbf{k}}^{2}=\left(1-\xi_{\mathbf{k}} / E_{\mathbf{k}}\right) / 2$, and $E_{\mathbf{k}}=$ $\sqrt{\xi_{\mathbf{k}}^{2}+\Delta^{2}}$. From the number constraint $n=2 \sum_{K} G(K)$, we can get the fermion number density

$$
n=2 \sum_{\mathbf{k}}\left[v_{\mathbf{k}}^{2}+f\left(E_{\mathbf{k}}\right) \frac{\xi_{\mathbf{k}}}{E_{\mathbf{k}}}\right],
$$

where $f(x)=1 /\left(e^{x / T}+1\right)$ is the Fermi distribution function.

Above $T_{c}$, the Thouless criterion should be modified by $U^{-1}+\chi(0)=a_{0} \mu_{p}$, where $\mu_{p}$ is the effective pair chemical potential and $a_{0}$ is the coefficient of the linear $\Omega$ term in the Taylor expansion of the inverse $T$-matrix (see below) [4]. This leads to the extended gap equation

$$
\frac{m}{4 \pi a}=\sum_{\mathbf{k}}\left[\frac{1}{2 \epsilon_{\mathbf{k}}}-\frac{1-2 f\left(E_{\mathbf{k}}\right)}{2 E_{\mathbf{k}}}\right]+a_{0} \mu_{p},
$$

with $\mu_{p}=0$ at $T \leq T_{c}$. Here, the coupling strength $U$ has been replaced by the $s$-wave scattering length $a$ via $U^{-1}=m / 4 \pi a-\sum_{\mathbf{k}} 1 / 2 \epsilon_{\mathbf{k}}$. Note that this scattering length is different from that defined in simple 3D free space, since $k_{z}$ is now restricted to within the first BZ. We caution that it does not necessarily yield the experimentally measured scattering length. One can define an effective scattering length 
via $a_{\text {eff }}=a / \sqrt{2 m t d}$, which is more comparable to the physical scattering length. For details, see Ref. [35] and its supplementary materials. It should also be noted that we have implicitly assumed a negative $U$ model for the lattice direction and the on-site $U$ is same as the in-plane pairing strength $U$. In real space, the pairing interaction is given by $U\left(\mathbf{r}, \mathbf{r}^{\prime}\right)=U \delta\left(x-x^{\prime}\right) \delta\left(y-y^{\prime}\right) \delta_{i j}$, where $i, j$ is the lattice site index in the $\hat{z}$ direction.

The inverse $T$-matrix expansion [4], after analytic continuation $\left(i \Omega_{l} \rightarrow \Omega+i 0^{+}\right)$, is given by

$$
t_{p g}^{-1}(\Omega, \mathbf{q}) \approx a_{1} \Omega^{2}+a_{0}\left(\Omega-\Omega_{\mathbf{q}}+\mu_{p}\right),
$$

with $\Omega_{\mathbf{q}}=B_{\|} \mathbf{q}_{\|}^{2}+2 t_{B}\left[1-\cos \left(q_{z} d\right)\right]$. Here $B_{\|}=1 / 2 M_{\|}$, with $M_{\|}$being the effective pair mass in the $x y$-plane, and $t_{B}$ is the effective hopping integral for noncondensed pairs. The $a_{1}$ term serves as a small quantitative correction; except in the weak coupling BCS regime, we have $a_{1} T_{c} \ll a_{0}$. The coefficients $a_{1}, a_{0}, B_{\|}$and $t_{B}$ can be derived from the pair susceptibility via straightforward Taylor expansion, as given in the Appendix. Consequently, we have the pseudogap equation

$$
a_{0} \Delta_{p g}^{2}=\sum_{\mathbf{q}} \frac{b\left(\tilde{\Omega}_{\mathbf{q}}\right)}{\sqrt{1+4 \frac{a_{1}}{a_{0}}\left(\Omega_{\mathbf{q}}-\mu_{p}\right)}}
$$

where $b(x)$ is the Bose distribution function and $\tilde{\Omega}_{\mathbf{q}}=$ $\left\{\sqrt{a_{0}^{2}\left[1+4 a_{1}\left(\Omega_{\mathbf{q}}-\mu_{p}\right) / a_{0}\right]}-a_{0}\right\} / 2 a_{1}$ is the pair dispersion. When $a_{1} / a_{0}$ is small, we have $\tilde{\Omega}_{\mathbf{q}}=\Omega_{\mathbf{q}}-\mu_{p}$. Then $a_{0} \Delta_{p g}^{2}$ yields the density of finite momentum pairs. Including the condensate, the total pair density is given by $n_{p}=a_{0} \Delta^{2}$.

Equations (2)-(5) form a closed set of self-consistent equations, which can be used to solve for $\left(\mu, T^{*}\right)$ with $\Delta=0$, for $\left(\mu, \Delta_{p g}, T_{c}\right)$ with $\Delta_{s c}=0$, and for $\left(\mu, \Delta, \Delta_{p g}\right)$ at $T<T_{c}$. Here the pair formation temperature $T^{*}$ is approximated by the mean-field $T_{c}$, and the order parameter $\Delta_{s c}$ can be derived from $\Delta_{s c}^{2}=\Delta^{2}-\Delta_{p g}^{2}$ below $T_{c}$.

\section{B. Asymptotic behavior in the deep BEC regime}

In the deep BEC regime, $\mu \rightarrow-\infty$. The integrals in the equations can be performed analytically using Taylor expansions. The fermion number equations reduce to

$$
n=-\frac{m \Delta^{2}}{4 \pi \mu d} \quad \text { or } \quad \Delta=\sqrt{\frac{4 \pi|\mu| d n}{m}} .
$$

With the help of Eq. (6), the chemical potential $\mu$ can be uniquely determined by the gap equations. Then $\mu$ and the gap $\Delta$ are given by

$$
\begin{aligned}
\mu & =-t e^{d / a}+2 t+\frac{2 \pi d n}{m} \\
\Delta & =2 \sqrt{\frac{\pi t d n}{m}} e^{d / 2 a}\left(1-\frac{\pi d n}{m t} e^{-d / a}\right) .
\end{aligned}
$$

Note that the exponential behavior of $\mu$ and $\Delta$ as a function of $1 / k_{F} a$ is an important feature of the quasi-two dimensionality of the continuum-lattice mixed system. This should be contrasted with the corresponding behaviors in the 3D continuum and 3D lattices, where power law dependencies are found. In particular, a 3D continuum has the scaling relation $\Delta \sim|\mu|^{1 / 4}$ in the BEC regime and thus $\Delta^{2} / \mu$ decreases with $1 / k_{F} a$. On the other hand, for a 3D lattice, due to the finite volume of the unit cell, both $|\mu|$ and $\Delta$ grow linearly with $|U|$, with a ratio of $\Delta /|\mu|=\sqrt{2 n-n^{2}} /(1-n)$ for $n<1$ per unit cell. In contrast, for the present continuum-lattice mixed system, the ratio $\Delta^{2} / \mu$ approaches a constant, independent of pairing strength. For this reason, the (2nd and 3rd) correction terms in Eq. (7) are also constants, independent of the interaction strength. The correction term in Eq. (8) quickly drops as $|U|$ increases.

To solve for $T_{c}$, we first derive the pair dispersion, and find

$$
\begin{aligned}
B_{\|} & =\frac{1}{4 m}, \\
t_{B} & =\frac{t^{2}}{2|\mu|} \approx \frac{t}{2} e^{-d / a} .
\end{aligned}
$$

While the in-plane pair mass in the BEC regime is given by $2 m$, as expected, the out-of-plane pair mass becomes exponentially heavy, as a function of increasing $d / a$. This can be easily understood since on a lattice pairs hop mainly via "virtual ionization" [36] (i.e., virtual pair unbinding) and thus its mobility is inversely proportional to the pair binding energy $2|\mu|$. The pseudogap equation now becomes the equation for pair density $n_{p}$,

$$
a_{0} \Delta^{2} \equiv n_{p}=\frac{n}{2},
$$

and the coefficient $a_{1}$ is given by

$$
a_{1} \Delta^{2}=-\frac{n}{8 \mu},
$$

which becomes exponentially small in the BEC limit. Now one readily derive the solution for $T_{c}$,

$$
T_{c}=\frac{2 \pi B_{\|} d n}{d / a-\ln \left(t / T_{c}\right)} \approx \frac{\pi a n}{2 m}=\frac{k_{F} a}{3 \pi} T_{F},
$$

where use has been made of the definition of $k_{F}=\left(3 \pi^{2} n\right)^{1 / 3}$ and $E_{F}=T_{F}=k_{F}^{2} / 2 m$ (as in 3D continuum) in the last step, and we have dropped the small logarithmic correction $\ln \left(t / T_{c}\right)$ in the denominator. An important and interesting aspect of this result is that the BEC asymptote is essentially independent of $d$, and the effect of $t$ only enters through a logarithmic correction, which can be safely neglected in the asymptote as well.

\section{Superfluid density}

Given the solution of the self-consistent equations, one can easily investigate the transport behavior of the system. As 
an example, in this subsection, we shall present calculations for superfluid "density" $n_{s} / m$, which is important quantity in the superfluid phase. In superconductors, it is often measured via the London penetration depth $\lambda_{L}$, especially at low $T$, with the relation $n_{s} / m \propto \lambda_{L}^{-2}$. The temperature dependence at low $T$ often serves as a strong indicator for the pairing symmetry of a superconductor, as it depends strongly on the pairing symmetry. BCS mean-field calculations show that it exhibits exponential $T$ dependence for an $s$-wave superconductor, and linear $T$ dependence for a nodal $d$-wave superconductor [25, 34, 37, 38].

The expression for superfluid density can be derived following Refs. [25, 39], using the linear response theory. More technical details can be found in Ref. [40]. For the present charge-neutral atomic gases, we only need to assume a fictitious vector potential, which can actually be realized experimentally via synthetic gauge fields.

Without imbalance, the superfluid density is given by

$$
\left(\frac{n_{s}}{m}\right)_{i}=2 \sum_{\mathbf{k}} \frac{\Delta_{s c}^{2}}{E_{\mathbf{k}}^{2}}\left[\frac{1-2 f\left(E_{\mathbf{k}}\right)}{2 E_{\mathbf{k}}}+f^{\prime}\left(E_{\mathbf{k}}\right)\right]\left(\frac{\partial \xi_{\mathbf{k}}}{\partial k_{i}}\right)^{2}
$$

where $i=x, y, z$ and $f^{\prime}(x)=-f(x) f(-x) / T$ is the directive of the Fermi distribution function.

Following Ref. [40], it can be shown that for the in-plane motion, $\left(n_{s} / m\right)_{\|}=\left(n_{s} / m\right)_{x}=\left(n_{s} / m\right)_{y}=n / m$ at $T=0$, since $\partial^{2} \xi_{\mathbf{k}} / \partial k_{i}^{2}=1 / m=$ const for $i=x, y$. In contrast, in the lattice direction, the inverse band mass $(1 / m)_{z}=$ $\partial^{2} \xi_{\mathbf{k}} / \partial k_{z}^{2}=2 t d^{2} \cos \left(k_{z} d\right)$ is $k_{z}$ dependent and scaled by the factor $t d^{2}$. As a consequence, we expect $\left(n_{s} / m\right)_{z} \propto t^{2} d^{2}$ and becomes small for realistic lattices, based on Eq. (14).

\section{NUMERICAL RESULTS AND DISCUSSIONS}

\section{A. Effect of lattice-continuum mixing on BCS-BEC crossover}

In this subsection, we first investigate the effect of latticecontinuum mixing on the behavior of $T_{c}$ and phase diagram throughout the BCS-BEC crossover regimes.

Shown in Fig. 1 is a typical phase diagram of a twocomponent balanced Fermi gas in a 1D optical lattice. Here we take realistic values for $t$ and $d$, with $t / E_{F}=0.05$ and $k_{F} d=2$. Note that in the zero lattice depth limit, the fermion energy in the lattice dimension should reduce to the simple parabolic dispersion, with mass $m$. Therefore, we set $t d^{2}<1 / 2 m$ as a constraint on the choice of the values of $t$ and $d$. Here we have $2 m t d^{2}=0.2$. The (yellow) shaded area is the superfluid phase, whereas the (blue) dashed curve is the mean-field solution of $T_{c}$. We take this as an estimate of the pair formation temperature, $T^{*}$. Between the $T^{*}$ and $T_{c}$ curves, there exists the pseudogap phase, where incoherent pairs exist but without phase coherence or Bose condensation. The $T_{c}$ curve reaches a maximum in the vicinity of unitarity, where $1 / k_{F} a=0$. In the BEC regime, $T_{c}$ decreases with increasing pairing strength. Note that the existence of the pseudogap phase is an inevitable feature of the BCS-BEC crossover.

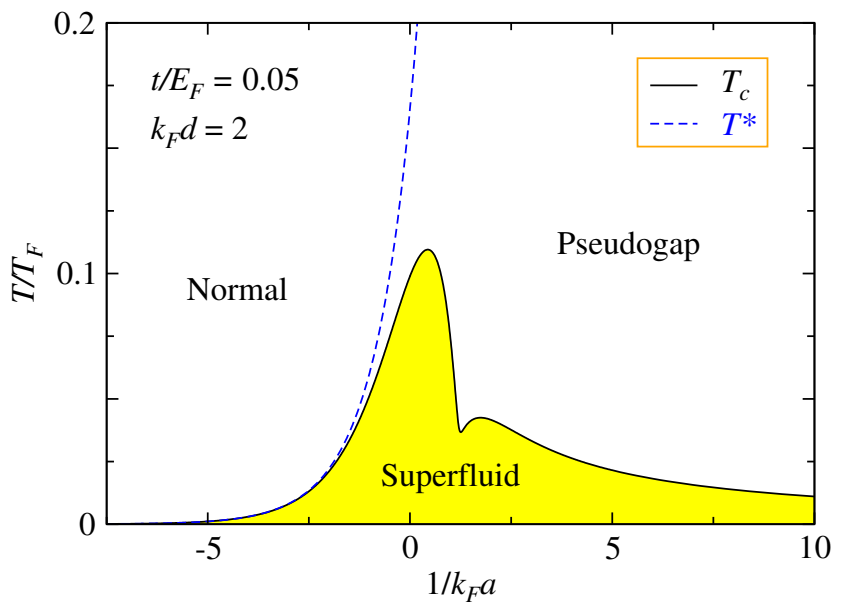

Figure 1. Typical phase diagram in the $T-1 / k_{F} a$ plane, calculated for $t / E_{F}=0.05$ and $k_{F} d=2$.

This phase diagram looks qualitatively similar to that in a 3D or quasi-2D pure lattice [15, 28]. However, we note that it in fact exhibits features of both pure $3 \mathrm{D}$ continuum and pure lattice cases. On the one hand, there is a minimum in $T_{c}$ around where the fermionic chemical potential $\mu$ changes sign, a feature of 3D continuum [15]. On the other hand, the decrease of $T_{c}$ with increasing $1 / k_{F} a$ in the BEC regime is a feature of pair hopping via virtual ionization [15, 36] in a lattice. The BEC regime is not accessible at high densities in a $3 \mathrm{D}$ or quasi-2D pure lattice. In a typical 3D lattice, the minimum disappears, leaving only a kink as a residue of the minimum [28]. In a quasi-2D lattice, such a minimum may exist only in the low density regime, where the inter-particle distance becomes much larger than the lattice constant. Indeed, the present system with an in-plane continuum space should be comparable to the low density limit when compared to the quasi-2D lattice case.

There are further distinctions between the present latticecontinuum mix and the pure systems. In Fig. 2. we show the comparison between the fully numerical and analytical solutions of (a) $T_{c}$ and (b) $\mu$ as a function of $1 / k_{F} a$ in the BEC regime. Shown in Fig. 2(a) are the $T_{c}$ curves in log-log scales for different values of $d$, while keeping $t$ fixed at $t=0.2 E_{F}$. Also shown is the analytically solution, Eq. (13), in the BEC regime (magenta dashed line). As is evident, all $T_{c}$ curves approach this $(t, d)$-independent analytical solution in the deep BEC regime. The larger $d$ case converges faster.

In Fig. 2(b), we present a semi-log plot of $-\mu$ and $\Delta$ as a function of $1 / k_{F} a$ for $t=0.05 E_{F}$ and $k_{F} d=2$, and compare the fully numerical solutions (solid lines) with the analytic expressions (dashed lines) given by Eqs. (7) and (8). As can be readily seen for the present case, the analytical expressions become a very good approximation for the fully numerical solutions for $1 / k_{F} a>3$.

From Fig. 2, we demonstrate that $T_{c}$ scales proportionally with $k_{F} a=\left(1 / k_{F} a\right)^{-1}$ in the BEC regime, following Eq. (13). This is different from its counterpart relation, $T_{c} \sim 1 / \bar{U}$, in a pure 3D lattice [15, 36]. While the general 

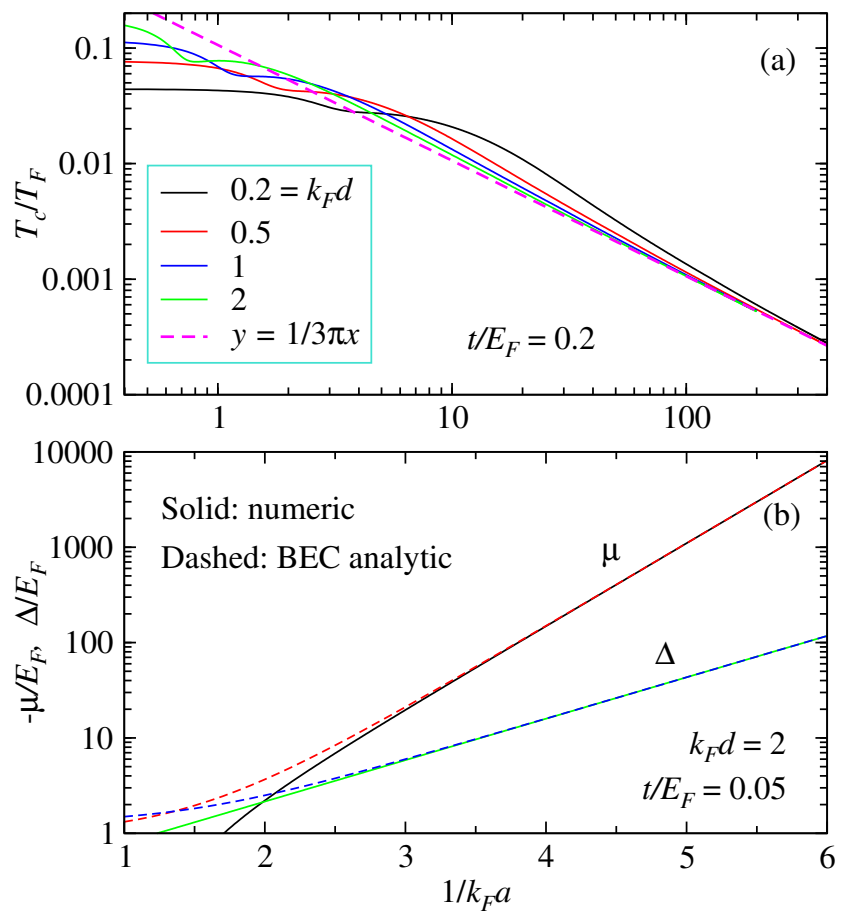

Figure 2. Comparison between fully numerical and analytical solutions in the BEC regime for (a) $T_{c}$ and (b) $\mu$, as a function of $1 / k_{F} a$. Shown in (a) are log-log plots of $T_{c}$ (solid lines) for $t / E_{F}=0.2$ and varying $k_{F} d$ from 0.2 to 2 , while the dashed line represents the analytical solution, Eq. (13). Plotted in (b) are $-\mu$ and $\Delta$ in a semi-log scale for $t / E_{F}=0.05$ and $k_{F} d=2$, where the analytical solutions (dashed lines) are given by Eqs. (7) and 8).

trend is the same, however, one does not have $1 / k_{F} a \propto U$ in the strong coupling limit.

The parameters $t$ and $d$ are the decisive factors for the shape of the Fermi surface. This can be seen from that of the lattice component in Fig. 1 of Ref. [29]. When $t$ is small, the first BZ of the lattice dimension will be fully occupied. In this case, a small $d$ means a large phase space $\pm \pi / d$ in the lattice direction, and therefore, will bring down the Fermi level as more particles now occupy the small $k_{\|}$but large $\left|k_{z}\right|$ states. On the contrary, a large $d$ will compress the phase space region between $\pm \pi / d$, and thus will push up the (in-plane) Fermi level. This can be understood from the real space perspective as well. As $d$ increases, the spacing between neighboring planes increases. Therefore, the area density within each plane has to increase accordingly in order to keep the overall average 3D density fixed. In this way, the Fermi level will be pushed up to $\mu_{0}=\sqrt{2 \pi n_{2 D}}$, where $n_{2 D}$ is the $2 \mathrm{D}$ fermion number density per plane. On the other hand, for a larger $t$, it may be possible that the first $\mathrm{BZ}$ in the $z$ direction is not fully occupied. The Fermi surface in the $z$ direction will allow a larger dispersion when $t$ increases. Depending on the size of $(t, d)$, the Fermi surface may possess a shape of an ellipsoid, a disc, a cylinder, or something in between. Except for the ellipsoid, all other types of Fermi surfaces are open. A van Hove singularity will appear at the Fermi level at the topological transition point between open and closed Fermi surfaces.
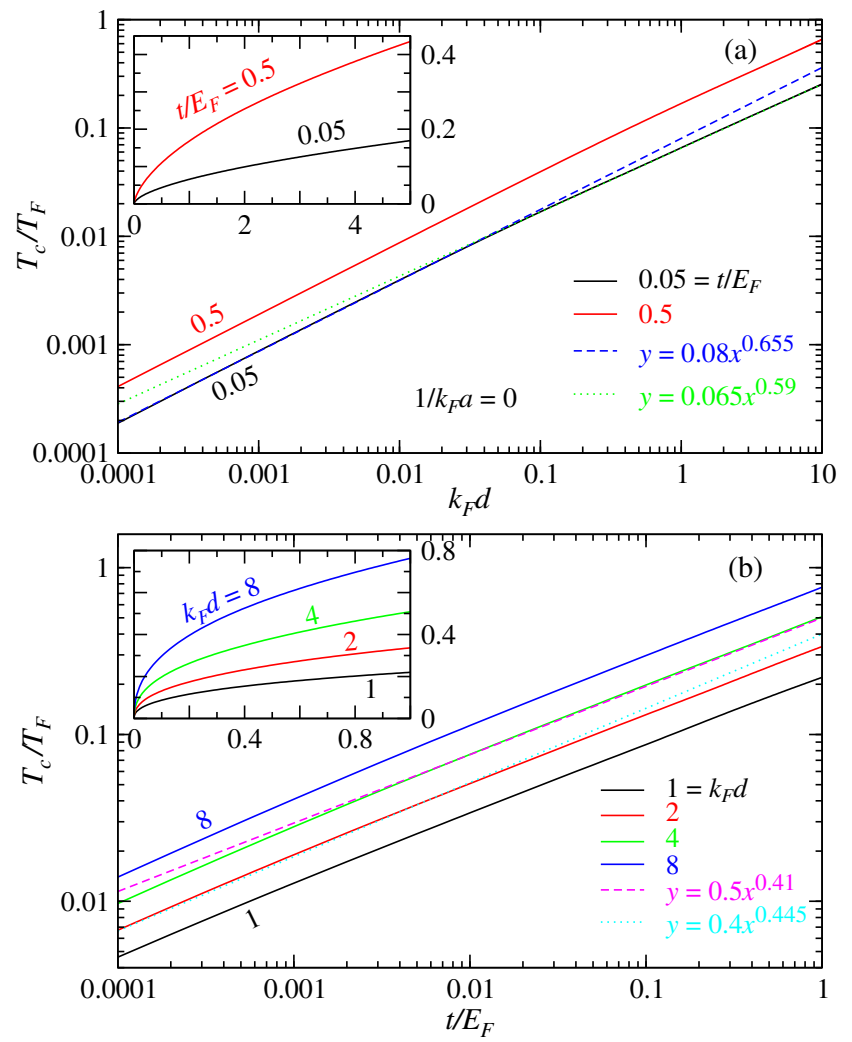

Figure 3. Behavior of $T_{c}$ at unitarity as a function of (a) $k_{F} d$ with $t / E_{F}=0.05$ (black lines) and 0.5 (red lines) and of (b) $t / E_{F}$ with $k_{F} d=1$ (black), 2 (red), 4 (green) and 8 (blue lines), respectively. Also shown in (a) are simple power laws, which fit the small (blue dashed) and large (green dotted) $d$ ranges well, respectively. Similarly, the cyan dashed and magenta dotted lines are simple power laws which fit the $T_{c}$ curves well in the large and small $t$ regimes, respectively.

Now we study the effect of $t, d$ on the behavior of $T_{c}$. First, we focus on the $T_{c}$ behavior at unitarity as a function of $t$ and $d$, since the unitary limit is a special point where the scattering length diverges, and thus the system may exhibit some universal behaviors.

Shown in Fig. 3 are log-log plots of $T_{c}$ as a function of (a) $d$ and (b) $t$, respectively. Their linear plots are given in the corresponding insets. Here we treat $t$ and $d$ as independent parameters, so that they may enter the experimentally inaccessible regime. Panel (a) covers a broad range of the $(t, d)$ parameter space, from large $t=0.5 E_{F}$ to small $t=0.05 E_{F}$, and from tiny $k_{F} d=0.0001$ to large $k_{F} d=10$. Surprisingly, $T_{c}$ exhibits a very good power law across such a big parameter space, with a scaling $T_{c} \propto d^{\alpha}$, where $\alpha$ is close to 0.655 for small $d$ and 0.59 for large $d$. Similarly, panel (b) also covers from $k_{F} d=1$ to 8 , and from $t / E_{F}=0.0001$ to 1.0 , and $T_{c}$ scales as $T_{c} \sim t^{\beta}$, where $\beta=0.445$ for small $t$ and 0.41 for large $t$. Overall, at unitarity, we have

$$
T_{c} \sim d^{\alpha} t^{\beta}, \quad \alpha=0.59 \sim 0.655, \beta=0.41 \sim 0.445 .
$$

Next, we show in Fig. 4 the $T_{c}$ curves throughout the entire 


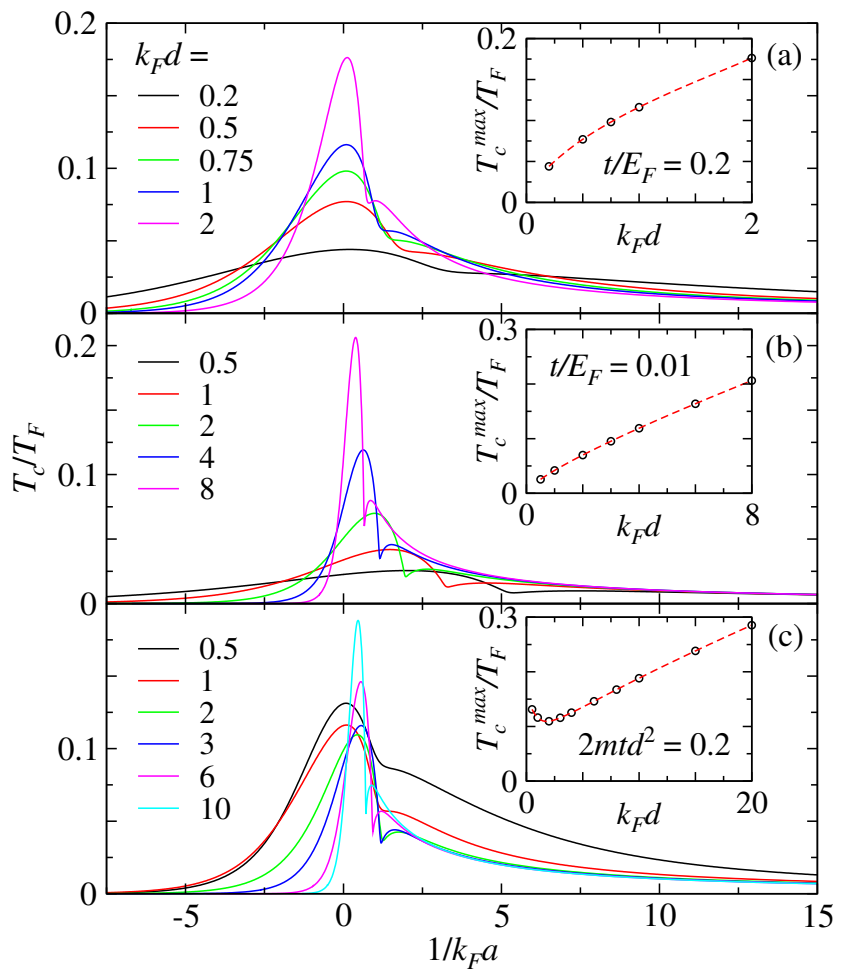

Figure 4. $T_{c}$ curves as a function of $1 / k_{F}$ a for different values of $k_{F} d$ at fixed (a) $t / E_{F}=0.2$, (b) 0.01 , and (c) $2 m t d^{2}=0.2$. The maximum $T_{c}$ near unitarity, $T_{c}^{\max }$, as a function of $d$, is plotted in the respective inset.

BCS-BEC crossover as a function of $1 / k_{F} a$ for different $t$ and $d$. Shown in panels (a) and (b) is $T_{c}$ for fixed $t / E_{F}=0.2$ and 0.01 , respectively, but with different values of $d$. Here we keep the product $t d^{2}<1 / 2 m$. As we can see, for fixed $t$, the maximum $T_{c}, T_{c}^{\max }$, increases with increasing $d$. At the same time, the entire $T_{c}$ curve is compressed horizontally towards unitarity, as $d$ increases. This is in accord with the exponential behavior of $\mu \sim-e^{d / a}$ in the BEC regime. We plot $T_{c}^{\max }$ versus $d$ in the corresponding insets, which exhibits a quasilinear behavior. The comparison between panels (a) and (b) for the same $d$ reveals that $T_{c}$ increases with $t$. Indeed, $T_{c}$ will be suppressed logarithmically to zero as $t$ approaches 0 [15]. We also note that the peak of maximum $T_{c}$ moves away from unitarity towards the BEC side as $d$ decreases. This also has to do with the exponential behavior of $\mu \sim-e^{d / a}$.

In Fig. 4 (c), we present the $T_{c}$ curves for fixed $2 m t d^{2}=0.2$ while changing $k_{F} d$ from 0.5 to 10 . Since $t$ decreases as $d$ increases, it is not surprising to see nonmonotonic behavior of $T_{c}^{\max }$ versus $d$, as shown in the inset. Nonetheless, we still see an overall increase of $T_{c}^{\max }$ with $d$ while keeping $t d^{2}$ fixed. This increase is not as dramatic as the fixed $t$ cases, reflecting the competing effects between increasing $d$ and decreasing $t$.

It should be pointed out that the increase of $T_{c}$ in Fig. 4 will disappear if we use the respective Fermi level $\mu_{0}$ in the noninteracting limit as the energy unit, as $\mu_{0}$ increases with $t$ and $d$ as well [41]. Nevertheless, this increase does make sense when one compares $T_{c}$ with the 3D homogeneous system of

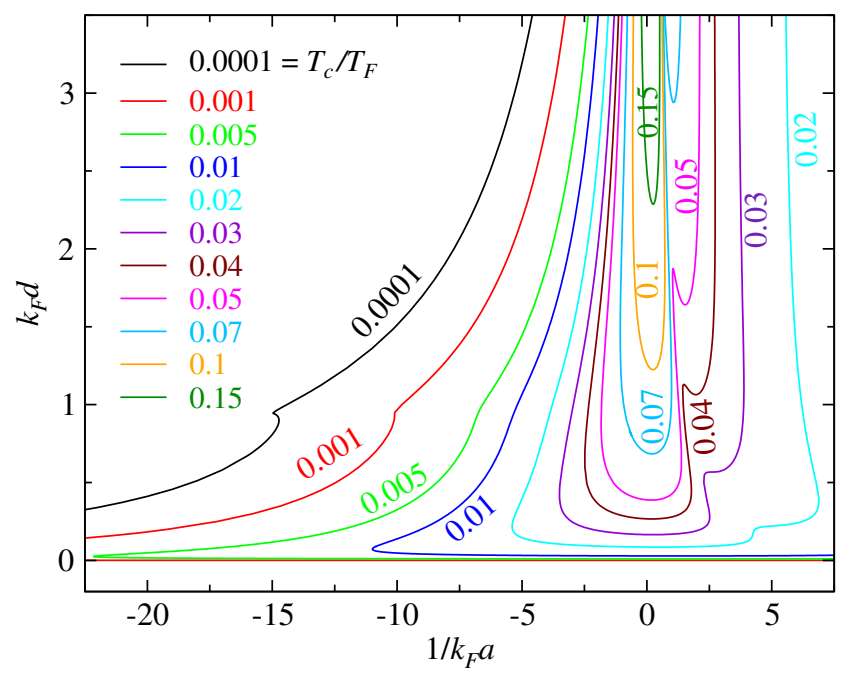

Figure 5. Contour plot of $T_{c} / T_{F}$ in the $k_{F} d-1 / k_{F} a$ plane for fixed $t / E_{F}=0.1$. The corresponding $T_{c}$ values are labeled near the curves.

the same fermion density.

With multiple tunable parameters, the complete superfluid phase diagram is very complex, occupying a hyper volume in the high dimensional phase space. We can show only hypersurfaces corresponding certain fixed parameters. As an example, presented in Fig. 5 are $T_{c}$ contours in the $k_{F} d-1 / k_{F} a$ plane with fixed $t / E_{F}=0.1$. From this figure, one can see that the highest $T_{c} \gtrsim 0.15$ is achieved at large $d$ near unitarity. The higher concentration of curves at large $d$ indicates that the $T_{c}$ curve is highly compressed towards unitarity as $d$ increases, as shown in Fig. 4. On the contrary, when $d$ becomes small $(\ll 1)$, the $T_{c}$ curve as a function of $1 / k_{F} a$ will be suppressed down and expanded along the $1 / k_{F} a$ axis. One can also consider a vertical cut at fixed $1 / k_{F} a$ in Fig. 5. A cut at $1 / k_{F} a=0$ will yield a curve as in the inset of Fig. $4(\mathrm{a}, \mathrm{b})$. The peak/dip structure of the $T_{c}$ contours at positive $1 / k_{F} a$ for $k_{F} d=0.03 \sim 0.07$ in Fig. 5 is associated with the dip near $\mu=0$ in the $T_{c}$ vs $1 / k_{F} a$ curves, as shown in Fig. 4. Another feature that is worth mentioning is the small kink in the contours on the BCS side, especially for the lowest $T_{c} / T_{F}=0.0001$. As can be seen, for all contours, this kink happens slightly below $k_{F} d=1$. For $t / E_{F}=0.1$, the topology of the Fermi surface changes from open to closed at $k_{F} d \approx 0.945$. The van Hove singularity associated with this topological change leads to logarithmic divergence of the density of states, and thus significantly enhances $T_{c}$, so that the $T_{c}$ contour will deform towards weaker pairing strength, as indeed shown by the low $T_{c}$ contours in Fig. 5. This singularity effect is washed out gradually by thermal broadening as $T$ increases. It becomes barely noticeable for $T_{c} / T_{F} \geq 0.01$. Note that the van Hove singularity effect on $T_{c}$ cannot readily be seen in other types of plot. 

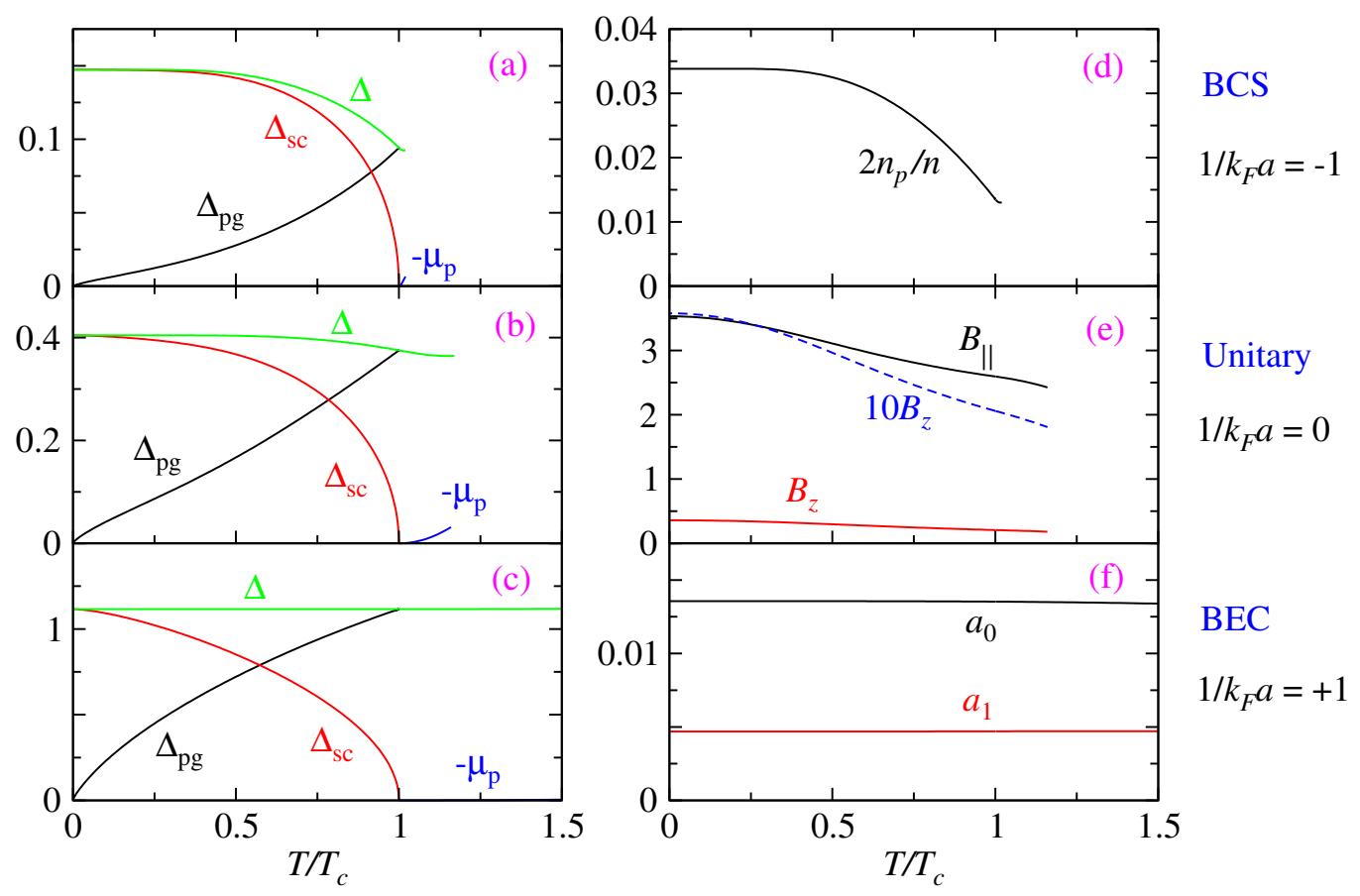

Figure 6. Behavior of the gaps and $-\mu_{p}$, as labeled, as a function of $T / T_{c}$, for (a) $1 / k_{F} a=-1$, (b) 0 , (c) +1 , with $T_{c} / T_{F}=0.07060$, 0.13135 and 0.05156 , for the BCS, unitary, and BEC regimes, respectively. Also plotted are (d) $2 n_{p} / n$ for $1 / k_{F} a=-1$, (e) $B_{\|}$and $B_{z}$ as well as $10 B_{z}$ for $1 / k_{F} a=0$, and (f) $a_{0}$ and $a_{1}$ for $1 / k_{F} a=+1$. Here $t / E_{F}=0.1$, and $k_{F} d=2$. Gaps and chemical potential are in units of $E_{F}$. The coefficients $B$ 's are in units of $1 / 2 m, a_{0}$ and $a_{1}$ in units of $k_{F}^{3} / E_{F}^{2}$ and $k_{F}^{3} / E_{F}^{3}$, respectively.

\section{B. Gaps in the superfluid phase}

In Fig. 6, we present, as an example, the behavior of the order parameter $\Delta_{s c}$ (red), the pseudogap $\Delta_{p g}$ (black) and the total gap $\Delta$ (green curves) and a few relevant quantities as a function of temperature in the superfluid phase. Also plotted is the solution slightly above $T_{c}$, especially for the pair chemical potential $\mu_{p}$. Shown in the figure is for the case of $k_{F} d=2, t / E_{F}=0.1$ for $1 / k_{F} a=-1,0$, and +1 , for the $\mathrm{BCS}$, unitary, and BEC regimes, respectively. There exists a pseudogap in all cases throughout the BCS-BEC crossover, as in the regular 3D continuum case [39]. The order parameter $\Delta_{s c}$ sets in at $T_{c}$ with decreasing $T$, while the pseudogap $\Delta_{p g}$ starts to decrease. The total gap increases with decreasing $T$ in the BCS regime, where $\Delta_{p g}$ is small, but stays roughly constant for the unitary and BEC cases. Above $T_{c}$, the pair chemical potential $\mu_{p}$ starts to decrease from 0 with increasing $T$. As seen in the figure, $-\mu_{p}$ increases much faster in the BCS than in the BEC regimes as a function of $T$ above $T_{c}$. This makes our simplified BCS form of the pseudogap self energy become quickly less accurate above $T_{c}$ in the BCS regime. The curves stop roughly where the approximation becomes inaccurate.

Figure 6(a) suggests that $-\mu_{p}$ increases linearly with $(T-$ $\left.T_{c}\right)$. Indeed, as one often finds in the weak fluctuation treatment in the framework of the mean-field BCS theory, $\mu_{p} \propto$ $-\left(T-T_{c}\right)$ above $T_{c}$ in the BCS limit [42, 43]. As the pairing becomes stronger, $\mu_{p}$ becomes quadratic in $\left(T-T_{c}\right)$, as manifested in Fig. 6(b). For the BEC case in Fig. 6(c), $-\mu_{p}$ stays small up to very high $T \gg T_{c}$. In this case, the gaps are large, and essentially all atoms form pairs, so that the system exhibits behaviors that are close to an ideal Bose gas.

We show in Fig. 6(d) the pair fraction for the BCS case, where the pairing is weak and the pair fraction is small. The temperature dependence of $n_{p}$ follows roughly that of $\Delta^{2}$ via Eq. (5), as $a_{0}$ is less sensitive to $T$. The pair density $n_{p}$ increases with $1 / k_{F} a$ and becomes $n / 2$ for $1 / k_{F} a=+1$, which has $\mu / E_{F} \approx-0.12<0$ for all $T \leq T_{c}$. For $B_{\|}$ and $B_{z}$, we show for the unitary case in Fig. 6(e). Their temperature dependencies are stronger in the $\mathrm{BCS}$ regime and weaker in the BEC regime. In addition, $B_{\|}$approaches $1 / 4 m$ in the BEC limit. At the same time, $B_{z}$ becomes exponentially smaller in the BEC regime, as given by Eq. (10). Finally, the $T$ dependencies of $a_{0}$ and $a_{1}$ are shown in Fig. 6 f) for the BEC case. Both $a_{0}$ and $a_{1}$ become essentially $T$ independent, as does the total gap. It is also evident that $a_{1} T_{c} \ll a_{0}$ for this case. The $a_{1}$ term in the inverse $T$-matrix expansion is quantitatively important only in the BCS regime, where we find $a_{1} T_{c} / a_{0} \sim 10$ for the case in Fig. 6.a). More detailed discussions of the influence of the $a_{1}$ term can be found in Ref. [40] for the somewhat similar 3D continuum case.

\section{Superfluid density}

Now we present the result for the superfluid density calculations. Shown in Fig 7 are the in-plane and lattice components of the superfluid density, from top to bottom, for the BCS, 

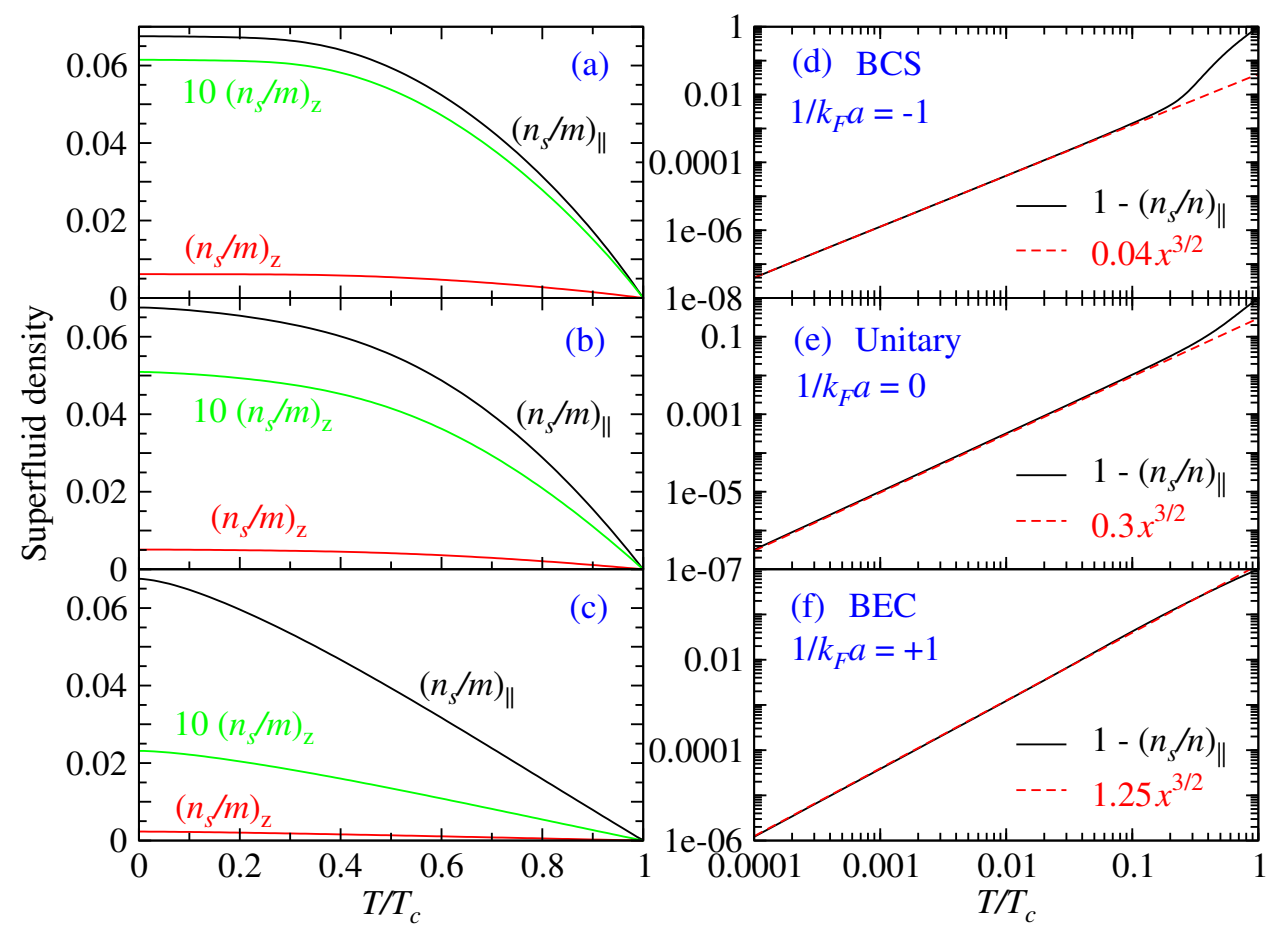

Figure 7. Behavior of the in-plane (black curves) and lattice components (red curves) of the superfluid densities, as labeled, as a function of $T / T_{c}$, for (a) $1 / k_{F} a=-1$, (b) 0 , (c) +1 , for the BCS, unitary, and BEC regimes, respectively, corresponding to Fig. 6 Also shown is $10\left(n_{s} / m\right)_{z}$ (green curves) for clarity. Plotted in (d-f) are the corresponding normal fluid fraction $1-\left(n_{s} / n\right)_{\|}$(black solid curves) in log-log scales, and simple $T^{3 / 2}$ power laws (red dashed lines) for comparison. The zero $T$ value of $\left(n_{s} / m\right)_{\|}$is given by $2 / 3 \pi^{2}$ in our convention of units.

unitary and BEC cases (of Fig. 6), respectively. The left column presents $\left(n_{s} / m\right)_{\|}$(black) and $\left(n_{s} / m\right)_{z}$ (red), as well as $10\left(n_{s} / m\right)_{z}$ (green curves). The right column presents the inplane normal fluid fraction, $1-\left(n_{s} / n\right)_{\|}$, as a function of $T / T_{c}$ in log-log scales (black solid lines). For comparison, we plot simple power laws of $\left(T / T_{c}\right)^{3 / 2}$ (red dashed lines), with different coefficients to fit roughly the corresponding solid lines. Here the message is clear. In the BCS case, the linear plot in Fig 7 (a) looks very much like an exponential $T$ dependence at low $T$. Only a log-log plot in Fig 7 (d) reveals that the leading dominant term is actually a $T^{3 / 2}$ power law. The small coefficient, 0.04 , in front of $\left(T / T_{c}\right)^{3 / 2}$, is consistent with the flatness of $\left(n_{s} / m\right)_{\|}$at low $T$ in Fig 7 (a). Nevertheless, the power law contributions from finite momentum pairs always dominate the exponentially activated term from Bogoliubov quasiparticles. As the pairing strength, or $1 / k_{F} a$, increases, the magnitude of the power law term becomes larger. For the unitary case, even in the linear plot in Fig $7(\mathrm{~b}),\left(n_{s} / m\right)_{\|}$deviates strongly from exponential behavior. The coefficient increases to 0.3, as shown in Fig. 7(e). For the BEC case in Fig 7 (c), the quasiparticle contributions become negligible, and $\left(n_{s} / m\right)_{\|}$becomes almost purely a $\left(T / T_{c}\right)^{3 / 2}$ power law. As one can see in Fig 7 (f), the dash line overlays with the solid curve essentially for the entire range of $T \leq T_{c}$. It should be noted, however, that the coefficient is now 1.25, larger than 1 . This reflects the fact that the system is quasi-2D rather than $3 \mathrm{D}$; a pure $\left(T / T_{c}\right)^{3 / 2}$ is only for a pure $3 \mathrm{D}$ case. Indeed, as one can see from Fig $7(\mathrm{c}),\left(n_{s} / m\right)_{\|}$becomes more of a straight line for the upper half of $T / T_{c}$, to be compatible with the larger-than-unity coefficient 1.25 . Theoretically, as $T$ becomes higher, more high in-plane momentum $q_{\|}$pairs will be excited, which can feel the quasi-two dimensionality.

The lattice component of the superfluid density, $\left(n_{s} / m\right)_{z}$, (red curves) in Fig 7(a-c) is substantially smaller than $\left(n_{s} / m\right)_{\|}$, as discussed earlier. Its temperature dependence is close to that of $\left(n_{s} / m\right)_{\|}$, as can be seen more clearly from the (green) $10 \times$ magnified curves. This is because both are mainly governed by the prefactor $\Delta_{s c}^{2}=\Delta^{2}-\Delta_{p g}^{2}$ in Eq. 14, and the second term, $\Delta_{p g}^{2} \propto T^{3 / 2}$, yields the $T^{3 / 2}$ power law for both components of $\left(n_{s} / m\right)$. For the present $s$-wave pairing, the rest of Eq. (14) yields an exponential $T$ dependence for the normal fluid, $e^{-\Delta / T}$, and thus becomes negligible at low $T$. It is also evident that $\left(n_{s} / m\right)_{z}$ decreases as the pairing becomes stronger toward BEC. This can be understood since $v_{\mathbf{k}}^{2}$ becomes more widespread in momentum space as $1 / k_{F} a$ increases, and thus pairs feel more strongly the effect of lattice momentum cutoff in the $z$ direction, so that the system becomes effectively more $2 \mathrm{D}$. On the other hand, the mobility of the pairs is controlled by $t_{B}$, which decreases rapidly with $1 / k_{F} a$. This determines the magnitude of $\left(n_{s} / m\right)_{z}$ in the $\mathrm{BEC}$ regime. 


\section{CONCLUSIONS}

In summary, we have studied the ultracold atomic Fermi gases in a $1 \mathrm{D}$ optical lattice with a pairing fluctuation theory, as they undergo a BCS-BEC crossover. We find that $T_{c}$ decreases with $1 / k_{F} a$ in the BEC regime and approaches asymptotically $T_{c} / T_{F}=\pi a n / 2 m$, which is independent of the lattice parameters $t$ and $d$. Both $|\mu|$ and the gap $\Delta$ grow exponentially as $e^{d / a}$ and $e^{d / 2 a}$, respectively, in the BEC regime so that the pair hopping integral $t_{B}$ decreases as $e^{-d / a}$. Moreover, the (maximum) $T_{c}$ near unitarity increases with both $t$ and $d$, with fractional power law exponents. On the BCS side, the effect of van Hove singularity on $T_{c}$ has been identified in the $T_{c}$ contours.

We find generally a pseudogap above and below $T_{c}$, away from the extreme BCS limit. While the total gap $\Delta$ is a smooth function across $T_{c}$, the order parameter sets in at $T_{c}$, and the pseudogap starts to decrease as $T$ decreases below $T_{c}$. Our calculated behavior of the pair chemical potential $\mu_{p}$ above $T_{c}$ are also in good agreement with existing literature. At low $T, \Delta_{p g}^{2} \sim T^{3 / 2}$. This leads to $T^{3 / 2}$ power laws for the low $T$ dependence of the superfluid density, despite that it looks visually like exponential in the BCS regime.

Our findings have not been reported in the literature. Although precise control and measurements of the gaps and superfluid density remains challenging experimentally at present, we believe that our predictions can be tested in future experiments.

\section{ACKNOWLEDGMENTS}

We thank the useful discussions with Chenchao $\mathrm{Xu}$, Lin Sun and Yanming Che. This work was supported by the NSF of China (Grant No. 11774309 and No. 11674283), and the NSF of Zhejiang Province of China (Grant No. LZ13A040001). C. Lee was supported by the NSF of China (Grants No. 11874434 and No. 11574405).

\section{Appendix A: Coefficients of the Taylor-expanded inverse $T$-matrix}

In this Appendix, we present concrete expressions for the coefficients of the Taylor expansion of the inverse $T$-matrix,
$t(\Omega, \mathbf{q})$, after analytical continuation,

$$
t_{p g}^{-1}(\mathbf{q}, \Omega)=a_{1} \Omega^{2}+a_{0}\left(\Omega-\Omega_{\mathbf{q}}+\mu_{p}+i \Gamma_{\mathbf{q}, \Omega}\right)
$$

Here $\mu_{p}=t^{-1}(0,0) / a_{0}$, which vanishes for $T \leq T_{c}$. In the long wavelength limit,

$$
\Omega_{\mathbf{q}}=B_{\|} q_{\|}^{2}+B_{z} q_{z}^{2} \equiv \frac{q_{\|}^{2}}{2 M_{\|}}+\frac{q_{z}^{2}}{2 M_{z}}
$$

with $B_{z}=t_{B} d^{2}$.

Before expansion, the inverse $T$ matrix is given by

$$
\begin{aligned}
t_{\mathbf{q}, \Omega+i 0^{+}}^{-1}= & U^{-1}+\sum_{\mathbf{k}}\left[\frac{1-f\left(E_{\mathbf{k}}\right)-f\left(\xi_{\mathbf{k}-\mathbf{q}}\right)}{E_{\mathbf{k}}+\xi_{\mathbf{k}-\mathbf{q}}-\Omega-i 0^{+}} u_{\mathbf{k}}^{2}\right. \\
& \left.-\frac{f\left(E_{\mathbf{k}}\right)-f\left(\xi_{\mathbf{k}-\mathbf{q}}\right)}{E_{\mathbf{k}}-\xi_{\mathbf{k}-\mathbf{q}}+\Omega+i 0^{+}} v_{\mathbf{k}}^{2}\right]
\end{aligned}
$$

Then we have

$$
\begin{aligned}
a_{0}= & \frac{1}{2 \Delta^{2}} \sum_{\mathbf{k}}\left[\left[1-2 f\left(\xi_{\mathbf{k}}\right)\right]-\frac{\xi_{\mathbf{k}}}{E_{\mathbf{k}}}\left[1-2 f\left(E_{\mathbf{k}}\right)\right]\right] \\
= & \frac{1}{2 \Delta^{2}}\left[n-2 \sum_{\mathbf{k}} f\left(\xi_{\mathbf{k}}\right)\right] \\
a_{1}= & \frac{1}{2 \Delta^{4}} \sum_{\mathbf{k}} E_{\mathbf{k}}\left[\left(1+\frac{\xi_{\mathbf{k}}^{2}}{E_{\mathbf{k}}^{2}}\right)\left[1-2 f\left(E_{\mathbf{k}}\right)\right]\right. \\
& \left.-2 \frac{\xi_{\mathbf{k}}}{E_{\mathbf{k}}}\left[1-2 f\left(\xi_{\mathbf{k}}\right)\right]\right]
\end{aligned}
$$

and the imaginary part

$$
\begin{aligned}
\Gamma_{\mathbf{q}, \Omega}= & \frac{\pi}{a_{0}} \sum_{\mathbf{k}}\left\{\left[1-f\left(E_{\mathbf{k}}\right)-f\left(\xi_{\mathbf{k}-\mathbf{q}}\right)\right] u_{\mathbf{k}}^{2} \delta\left(E_{\mathbf{k}}+\xi_{\mathbf{k}-\mathbf{q}}-\Omega\right)\right. \\
& \left.+\left[f\left(E_{\mathbf{k}}\right)-f\left(\xi_{\mathbf{k}-\mathbf{q}}\right)\right] v_{\mathbf{k}}^{2} \delta\left(E_{\mathbf{k}}-\xi_{\mathbf{k}-\mathbf{q}}+\Omega\right)\right\} . \quad(\mathrm{A6})
\end{aligned}
$$

We have $\Gamma_{\mathbf{q}, \Omega}=0$ when $-\left(E_{\mathbf{k}}-\xi_{\mathbf{k}-\mathbf{q}}\right)_{\min }<\Omega_{\mathbf{q}}<\left(E_{\mathbf{k}}+\right.$ $\left.\xi_{\mathbf{k}-\mathbf{q}}\right)_{\min }$, and in general $\Gamma_{\mathbf{q}, \Omega}$ is much smaller than $\Omega_{\mathbf{q}}$ for small $q$ at $T \leq T_{c}$. For details, see Ref. [40].

The pair dispersion coefficients are given by

$$
\begin{aligned}
B_{i}= & \left.\frac{1}{2} \frac{\partial^{2} \Omega_{\mathbf{q}}}{\partial q_{i}^{2}}\right|_{\mathbf{q}=\mathbf{0}} \\
= & -\frac{1}{2 a_{0} \Delta^{2}} \sum_{\mathbf{k}}\left\{\left[2 f^{\prime}\left(\xi_{\mathbf{k}}\right)+\frac{E_{\mathbf{k}}}{\Delta^{2}}\left[\left(1+\frac{\xi_{\mathbf{k}}^{2}}{E_{\mathbf{k}}^{2}}\right)\left[1-2 f\left(E_{\mathbf{k}}\right)\right]-2 \frac{\xi_{\mathbf{k}}}{E_{\mathbf{k}}}\left[1-2 f\left(\xi_{\mathbf{k}}\right)\right]\right]\right]\left(\frac{\partial \xi_{\mathbf{k}}}{\partial k_{i}}\right)^{2}\right. \\
& \left.-\frac{1}{2}\left[\left[1-2 f\left(\xi_{\mathbf{k}}\right)\right]-\frac{\xi_{\mathbf{k}}}{E_{\mathbf{k}}}\left[1-2 f\left(E_{\mathbf{k}}\right)\right]\right] \frac{\partial^{2} \xi_{\mathbf{k}}}{\partial k_{i}^{2}}\right\}
\end{aligned}
$$

Given the dispersion $\xi_{\mathbf{k}}=\frac{k_{\|}^{2}}{2 m}-2 t\left[1-\cos \left(k_{z} d\right)\right]-\mu$ for

1DOL, we have, for $i=x, y$,

$$
\left(\frac{\partial \xi_{\mathbf{k}}}{\partial k_{i}}\right)^{2}=\frac{k_{i}^{2}}{m^{2}}, \quad \frac{\partial^{2} \xi_{\mathbf{k}}}{\partial k_{i}^{2}}=\frac{1}{m},
$$


and for $i=z$,

$$
\left(\frac{\partial \xi_{\mathbf{k}}}{\partial k_{z}}\right)^{2}=(2 t d)^{2} \sin ^{2}\left(k_{z} d\right), \quad \frac{\partial^{2} \xi_{\mathbf{k}}}{\partial k_{z}^{2}}=2 t d^{2} \cos \left(k_{z} d\right) .
$$

[1] M. Köhl, H. Moritz, T. Stöferle, K. Günter, and T. Esslinger, Fermionic atoms in a three dimensional optical lattice: Observing Fermi surfaces, dynamics, and interactions, Phys. Rev. Lett. 94, 080403 (2005).

[2] I. Bloch, Ultracold quantum gases in optical lattices, Nat. Phys. 1, $23(2005)$

[3] A. Georges, Condensed matter physics with light and atoms: Strongly correlated cold fermions in optical lattices, in Ultracold Fermi Gases, Proceedings of the International School of Physics "Enrico Fermi", Vol. CLXIV, edited by M. Inguscio, W. Ketterle, and C. Salomon, Società Italiana di Fisica Bologna, Italy (IOS Press, Amsterdam, 2008) pp. 477-510.

[4] Q. J. Chen, J. Stajic, S. N. Tan, and K. Levin, BCS-BEC crossover: From high temperature superconductors to ultracold superfluids, Phys. Rep. 412, 1 (2005)

[5] I. Bloch, J. Dalibard, and W. Zwerger, Many-body physics with ultracold gases, Rev. Mod. Phys. 80, 885 (2008)

[6] S. Giorgini, L. P. Pitaevskii, and S. Stringari, Theory of ultracold atomic Fermi gases, Rev. Mod. Phys. 80, 1215 (2008).

[7] A. Cichy and R. Micnas, The spin-imbalanced attractive Hubbard model in $\mathrm{d}=3$ : Phase diagrams and BCS-BEC crossover at low filling, Ann. Phys. 347, 207 (2014)

[8] X.-W. Guan, M. T. Batchelor, and C. Lee, Fermi gases in one dimension: From Bethe ansatz to experiments, Rev. Mod. Phys. 85, 1633 (2013)

[9] P. Dyke, E. D. Kuhnle, S. Whitlock, H. Hu, M. Mark, S. Hoinka, M. Lingham, P. Hannaford, and C. J. Vale, Crossover from $2 \mathrm{D}$ to $3 \mathrm{D}$ in a weakly interacting Fermi gas, Phys. Rev. Lett. 106, 105304 (2011)

[10] A. T. Sommer, L. W. Cheuk, M. J. H. Ku, W. S. Bakr, and M. W. Zwierlein, Evolution of fermion pairing from three to two dimensions, Phys. Rev. Lett. 108, 045302 (2012)

[11] M. G. Ries, A. N. Wenz, G. Zürn, L. Bayha, I. Boettcher, D. Kedar, P. A. Murthy, M. Neidig, T. Lompe, and S. Jochim, Observation of pair condensation in the quasi-2d bec-bcs crossover, Phys. Rev. Lett. 114, 230401 (2015)

[12] P. A. Murthy, I. Boettcher, L. Bayha, M. Holzmann, D. Kedar, M. Neidig, M. G. Ries, A. N. Wenz, G. Zürn, and S. Jochim, Observation of the berezinskii-kosterlitz-thouless phase transition in an ultracold fermi gas, Phys. Rev. Lett. 115, 010401 (2015)

[13] M. Feld, B. Froehlich, E. Vogt, M. Koschorreck, and M. Koehl, Observation of a pairing pseudogap in a two-dimensional Fermi gas, Nature 480, 75 (2011)

[14] R. Micnas, J. Ranninger, and S. Robaszkiewicz, Superconductivity in narrow-band systems with local nonretarded attractive interactions., Rev. Mod. Phys. 62, 113 (1990)

[15] Q. J. Chen, I. Kosztin, B. Jankó, and K. Levin, Superconducting transitions from the pseudogap state: $d$-wave symmetry, lattice, and low-dimensional effects., Phys. Rev. B 59, 7083 (1999)

[16] W. Hofstetter, J. I. Cirac, P. Zoller, E. Demler, and M. D. Lukin, High-temperature superfluidity of fermionic atoms in optical lattices., Phys. Rev. Lett. 89, 220407 (2002), W. V. Liu, F. Wilczek, and P. Zoller, Spin-dependent hubbard model and a quantum phase transition in cold atoms, Phys. Rev. A 70,
033603 (2004), M. A. Cazalilla, A. F. Ho, and T. Giamarchi, Two-component Fermi gas on internal-state-dependent optical lattices, Phys. Rev. Lett. 95, 226402 (2005), T. Koponen, J. Kinnunen, J.-P. Martikainen, L. M. Jensen, and P. Törmä, Fermion pairing with spin-density imbalance in an optical lattice, New J. Phys. 8, 179 (2006), A. Moreo and D. Scalapino, Cold attractive spin polarized fermi lattice gases and the doped positive u hubbard model, Phys. Rev. Lett. 98, 216402 (2007)

[17] C.-C. Chien, Y. He, Q. J. Chen, and K. Levin, Superfluidinsulator transitions at noninteger filling in optical lattices of fermionic atoms, Phys. Rev. A 77, 011601 (2008), C.-C. Chien, Q. J. Chen, and K. Levin, Fermions with attractive interactions on optical lattices and implications for correlated systems, Phys. Rev. A 78, 043612 (2008)

[18] M. Iskin and C. J. Williams, Population-imbalanced fermions in harmonically trapped optical lattices, Phys. Rev. A 78, 011603 (2008) Z. Cai, Y. Wang, and C. Wu, Stable fulde-ferrell-larkinovchinnikov pairing states in $2 \mathrm{~d}$ and $3 \mathrm{~d}$ optical lattices, Phys. Rev. A 83, 063621 (2011); T. Gottwald and P. G. J. van Dongen, Ground state properties of an asymmetric hubbard model for unbalanced ultracold fermionic quantum gases, Eur. Phys. J. B 61, 277 (2008), Y. Chen, Z. D. Wang, F. C. Zhang, and C. S. Ting, Exploring exotic superfluidity of polarized ultracold fermions in optical lattices, Phys. Rev. B 79, 054512 (2009), B. Wang, H.-D. Chen, and S. D. Sarma, Quantum phase diagram of fermion mixtures with population imbalance in onedimensional optical lattices, ibid. 79, 051604(R) (2009); Y. L. Loh and N. Trivedi, Detecting the elusive larkin-ovchinnikov modulated superfluid phases for imbalanced fermi gases in optical lattices, Phys. Rev. Lett. 104, 165302 (2010), X. Cui and Y. Wang, Polarized fermi gases in asymmetric optical lattices, Phys. Rev. A. 81, 023618 (2010).

[19] A.-H. Chen and G. Xianlong, Pure fulde-ferrell-larkinovchinnikov state in optical lattices, Phys. Rev. B 85, 134203 (2012) R. Mendoza, M. Fortes, M. A. Solís, and Z. Koinov, Superfluidity of a spin-imbalanced fermi gas in a threedimensional optical lattice, Phys. Rev. A 88, 033606 (2013)

[20] M. O. J. Heikkinen, D.-H. Kim, M. Troyer, and P. Törmä, Nonlocal quantum fluctuations and fermionic superfluidity in the imbalanced attractive hubbard model, Phys. Rev. Lett. 113, 185301 (2014), R. Peters and J. Bauer, Local origin of the pseudogap in the attractive hubbard model, Phys. Rev. B 92, 014511 (2015), S. Kitamura and H. Aoki, $\eta$-pairing superfluid in periodically-driven fermionic hubbard model with strong attraction, Phys. Rev. B 94, 174503 (2016)

[21] S.-J. Gu, R. Fan, and H.-Q. Lin, Ground state of a mixture of two species of fermionic atoms in a one-dimensional optical lattice, Phys. Rev. B 76, 125107 (2007), A. E. Feiguin and F. Heidrich-Meisner, Pairing states of a polarized Fermi gas trapped in a one-dimensional optical lattice, Phys. Rev. B 76, 220508(R) (2007), M. Rizzi, M. Polini, M. Cazalilla, M. Bakhtiari, M. Tosi, and R. Fazio, Fulde-Ferrell-LarkinOvchinnikov superfluidity in one-dimensional optical lattices, Phys. Rev. B 77, 245105 (2008); M. R. Bakhtiari, M. J. Leskinen, and P. Törmä, Spectral signatures of the Fulde-Ferrell- 
Larkin-Ovchinnikov order parameter in one-dimensional optical lattices, Phys. Rev. Lett. 101, 120404 (2008), T. Roscilde, C. D. E. Boschi, and M. Dalmonte, Pairing, crystallization and string correlations of mass-imbalanced atomic mixtures in onedimensional optical lattices, Europhys. Lett. 97, 23002 (2012), V. V. Franca, D. Hördlein, and A. Buchleitner, Fulde-FerrellLarkin-Ovchinnikov critical polarization in one-dimensional fermionic optical lattices, Phys. Rev. A 86, 033622 (2012)

[22] P. Fulde and R. A. Ferrell, Superconductivity in a strong spinexchange field, Phys. Rev. 135, A550 (1964)

[23] A. I. Larkin and Y. N. Ovchinnikov, Inhomogeneous state of superconductors, Sov. Phys. JETP 20, 762 (1965), [Zh. Eksp. Teor. Fiz. 47, 1136 (1964)].

[24] J. P. A. Devreese, S. N. Klimin, and J. Tempere, Resonant enhancement of the Fulde-Ferell-Larkin-Ovchinnikov state in three dimensions by a one-dimensional optical potential, Phys. Rev. A 83, 013606 (2011), J. P. A. Devreese, M. Wouters, and J. Tempere, Controlling the pair momentum of the fuldeferrell-larkin-ovchinnikov state in a three-dimensional fermi gas through a one-dimensional periodic potential, Phys. Rev. A 84, 043623 (2011), J. P. A. Devreese, S. Klimin, M. Wouters, and J. Tempere, The Fulde-Ferell-Larkin-Ovchinnikov state in a $3 \mathrm{~d}$ Fermi gas subjected to a 1D periodic potential, Mod. Phys. Lett. B 26, 1230014 (2012)

[25] Q. J. Chen, I. Kosztin, B. Jankó, and K. Levin, Pairing fluctuation theory of superconducting properties in underdoped to overdoped cuprates., Phys. Rev. Lett. 81, 4708 (1998)

[26] Q. J. Chen, Y. He, C.-C. Chien, and K. Levin, Theory of superfluids with population imbalance: Finite-temperature and BCSBEC crossover effects, Phys. Rev. B 75, 014521 (2007)

[27] Q. J. Chen, Y. He, C.-C. Chien, and K. Levin, Theory of radio frequency spectroscopy experiments in ultracold fermi gases and their relation to photoemission experiments in the cuprates, Rep. Prog. Phys. 72, 122501 (2009).

[28] Q. J. Chen, Zero-density-limit extrapolation of the superfluid transition temperature in a unitary atomic fermi gas on a lattice, Phys. Rev. A 86, 023610 (2012).

[29] L. F. Zhang, Y. M. Che, J. B. Wang, and Q. J. Chen, Exotic superfluidity and pairing phenomena in atomic Fermi gases in mixed dimensions, Sci. Rep. 7, 12948 (2017)

[30] Q. J. Chen and J. B. Wang, Pseudogap phenomena in ultracold atomic Fermi gases, Front. Phys. 9, 539 (2014)
[31] E. J. Mueller, Review of pseudogaps in strongly interacting Fermi gases, Rep. Prog. Phys. 80, 104401 (2017)

[32] A. L. Fetter and J. D. Walecka, Quantum Theory of ManyParticle Systems (McGraw-Hill, San Francisco, 1971).

[33] D. J. Thouless, Ann. Phys. 10, 553 (1960).

[34] T. Timusk and B. Statt, The pseudogap in high-temperature superconductors: An experimental survey., Rep. Prog. Phys. 62, 61 (1999)

[35] Q. J. Chen, J. B. Wang, and Y. Yu, Unusual destruction and enhancement of superfluidity of atomic Fermi gases by population imbalance in a one-dimensional optical lattice, arXiv:1904.09576.

[36] P. Nozières and S. Schmitt-Rink, Bose condensation in an attractive fermion gas: from weak to strong coupling superconductivity, J. Low Temp. Phys. 59, 195 (1985)

[37] J. R. Schrieffer, Theory of Superconductivity, 3rd ed. (Perseus Books, Reading, MA, 1983).

[38] J. F. Annett, N. Goldenfeld, and A. J. Leggett, Experimental constraints on the pairing state of the cuprate superconductors: An emerging consensus, in Physical Properties of High Temperature Superconductors $V$ edited by D. M. Ginsberg (World Scientific, Singapore, 1996) pp. 375-461, see also arXiv:condmat/9601060.

[39] I. Kosztin, Q. J. Chen, B. Jankó, and K. Levin, Relationship between the pseudo- and superconducting gaps: Effects of residual pairing correlations below $T_{c}$., Phys. Rev. B 58, R5936 (1998)

[40] Q. J. Chen, Generalization of BCS theory to short coherence length superconductors: A BCS-Bose-Einstein crossover scenario, Ph.D. thesis, University of Chicago (2000), available as arXiv:1801.06266.

[41] L. F. Zhang, J. B. Wang, Y. Yu, and Q. J. Chen, Ultra high temperature superfluidity in ultracold atomic Fermi gases with mixed dimensionality, Sci. China - Phys. Mech. Astron. 63, 227421 (2020) arXiv:1807.05049.

[42] A. Larkin and A. Varlamov, Theory of Fluctuations in Superconductors, International Series of Monographs on Physics (OUP Oxford, 2009).

[43] R. Boyack, Q. J. Chen, A. A. Varlamov, and K. Levin, Cuprate diamagnetism in the presence of a pseudogap: Beyond the standard fluctuation formalism, Phys. Rev. B 97, 064503 (2018) 